\title{
CONSUMO DE DROGAS PSICOACTIVAS Y FACTORES DE RIESGO FAMILIAR EN ADOLESCENTES ${ }^{1}$
}

\author{
PSYCHOACTIVE SUBSTANCE USE AND FAMILY RISK FACTORS IN ADOLESCENTS \\ Gregoria Francisca Canales Quezada², Thelma Díaz de Paredes, \\ Ana Carolina Guidorizzi Zanetti ${ }^{4}$, Carla Aparecida Arena Ventura ${ }^{5}$
}

\section{RESUMEN}

Introducción: Los adolescentes constituyen un grupo vulnerable de la población para el uso de drogas, siendo influenciados pór factores de riesgo de naturaleza biológica, psicológica o social, en el contexto individual, familiar y social. Los factores de riesgo familiar para el uso de drogas se relacionan con los estilos parentales de manejo familiar, incluyendo el tipo de comunicación construida, el establecimiento de normas y el manejo de sanciones, el clima emocional familiar y la cultura en torno a las drogas. Objetivo: Describir el consumo de drogas y los factores de riesgo familiar en los adolescentes del Reparto Primero de Mayo de la ciudad de León, Nicaragua. Materiales y Métodos: Estudio descriptivo y transversal con una muestra de 76 adolescentes, elegidos a través del muestreo probabilístico aleatorio simple. Para la obtención de los datos se aplicó un instrumento estructurado con preguntas cerradas. Resultados: Los factores de riesgo familiares presentes en la mayoría de los adolescentes fueran ausencia de los padres $65 \%$, caracterizada por la disfunción familiar, presencia de problemas en el manejo de la familia y relaciones conflictivas. De los 76 adolescentes, 53\% consumen alcohol y tabaco y el $47 \%$ consumen algún tipo de droga. Discusión y Conclusiones: De acuerdo al estudio son necesarios nuevos estudios en esta temática con mayor análisis que permitan continuar profundizando sobre el fenómeno de las drogas en este grupo vulnerable y el desarrollo de estrategias que contribuyan a la efectiva inserción social de los adolescentes y sus familiares. (Rev Cuid 2012; 3(3):260-9).

Palabras clave: Adolescente, Factores de Riesgo, Drogas. (Fuente: DeCS BIREME).

\section{ABSTRACT}

Introduction: The adolescents are a vulnerable group of the population regarding the use of drugs and are influenced by biological, psychological and social risk factors, in personal, family and social contexts. Family risk factors to the use of drugs are related to parents style in dealing with the children, including the type of communication, the establishment of rules and sanctions, the family emotional climate and the culture regarding drugs. Objective: Describe the use of drugs and the family risk factors in adolescents from the Reparto Primero de Mayo de la in the city of León, Nicaragua. Materials and Methods: Descriptive transversal study with a sample of 76 adolescents; selected through simple random probability sampling. In order to collect data, a structured instrument with closed questions was applied. Results: Family risks present in most adolescents, was the lack of living with the family $(65 \%)$, problems with family management and conflict relationships. Considering the 76 subjects, $53 \%$ use alcohol and tobacco and $47 \%$ use some type of drug. Discussion and Conclusions: Therefore, there is a need for other studies on this theme to deepen knowledge on drugs regarding this vulnerable group as well as the development of strategies to contribute to the social insertion of these adolescents and their families.

Key words: Adolescent, Risk Factors, Drugs. (Source: DeCS BIREME).

1 Articulo Original de Investigación.

2 Enfermera. Profesora de la Escuela de Enfermería - Universidad Nacional Autónoma de Nicaragua - León, Nicaragua.

3 Maestra. Enfermera de la Escuela de Enfermería - Universidad Nacional Autónoma de Nicaragua - León, Nicaragua.

4 Doctora. Docente de la Escuela de Enfermería de Ribeirão Preto de la Universidad de São Paulo - EERP-USP, Ribeirão Preto Brasil. Centro Colaborador de la OMS para el Desarrollo de la Investigación en Enfermería.

5 Profesor Asociado de la Escuela de Enfermería de Ribeirão Preto de la Universidad de São Paulo-EERP-USP, Ribeirão Preto Brasil. Centro Colaborador de la OMS para el Desarrollo de la Investigación en Enfermería. Correspondencia: Dirección: Avenida dos Bandeirantes, 3900. Campus Universitario. CEP 14040-902. Ribeirão Preto - SP, Brasil. Teléfono: +55 163602 3422. Fax: + 551636020518 . E-mail: caaventu@eerp.usp.br 


\section{INTRODUCCIÓN}

El problema mundial de las drogas psicoactivas constituye un fenómeno complejo, dinámico y multicausal que impone desafíos a los Estados y a sus gobiernos. Este problema representa un reto de alcance global que incide negativamente en la salud pública, la seguridad y el bienestar de toda la humanidad (1).

El Informe de la Organización Mundial de Salud, estableció que el 8,9\% de la carga total de morbilidad se atribuye al uso de sustancias psicoactivas. En muchos países, el impacto del uso y dependencia de sustancias psicoactivas se relaciona con un amplio conjunto de problemas de salud y de exclusión social, que contribuyen a la expansión de la carga de morbilidad, entre los cuales resalta el aumento del VIH/SIDA a través del uso de drogas intravenosas $(2,3)$.

Según reporte de la Oficina de las Naciones Unidas sobre Drogas y Delito -UNODC, cerca de 200 millones de personas, equivalente al $5 \%$ de la población mundial entre 15-64 años, han utilizado drogas por lo menos una vez durante los últimos 12 meses, del 2007 (4).

El fenómeno del consumo de drogas constituye así, un hecho complejo que abarca aspectos tan diversos y variados como los personales, familiares y sociales, con gran incidencia en la salud, la educación y la seguridad pública. Dicho fenómeno es un problema que se ha extendido a gran parte de la población de los países latinoamericanos, afectando especialmente a los adolescentes, mostrando índices crecientes en los niveles de consumo de esta población (5-11).

En este escenario, Nicaragua, como parte de un mundo globalizado, no es ajena al consumo epidémico de sustancias psicoactivas, que han venido modificando y transformando formas de vivir y de comportarse, que terminan muchas veces cobrando vidas humanas (8).

Estudio realizado en estudiantes de la Universidad de León, Nicaragua, sobre policonsumo de drogas reveló que el $52.1 \%$ ha utilizado droga algunas vez en su vida y la edad media para el consumo ha sido de 15.5 años (8). Otra investigación desarrollada en 2005 en 142 escuelas de primaria y 110 de secundaria en Managua, Nicaragua verificó que el "bañados" es la nueva droga que está consumiéndose en las escuelas. Se trata de la combinación de marihuana y crack, un poderoso estupefaciente que crea mayor adicción y puede causar más rápido la muerte por una sobredosis (12).

Otro estudio realizado en Nicaragua en relación a la prevalencia del consumo de drogas en los casos atendidos por lesiones y necropsias, se encontró que el grupo etáreo predominante fue el de 16 a 24 años (78 casos) de los cuales el $79.5 \%$ eran masculinos. También, $70.1 \%$ de los casos de necropsias fueron positivos en la prueba de alcohol, al $22.8 \%$ de cocaína, el $20.3 \%$ marihuana y el $2 \%$ por psicofármacos (13).

En este sentido, cabe destacar que la adolescencia es un período que se caracteriza por tratar de independizarse de los padres y demás adultos, estrechar la amistad con otros jóvenes y experimentar con muchas ideas, productos y estilos de vida. A veces entraña asumir mayores riesgos, adoptar decisiones, aceptar soluciones de compromiso y aprovechar oportunidades que podrían conducir a resultados inciertos. De esta forma, el consumo de drogas, tabaco y alcohol entre los adolescentes se está convirtiendo en una vía para escapar de situaciones de las que suelen sentirse impotentes para cambiar (14-17).

Para algunos adolescentes, experimentar el consumo de drogas es rito necesario para atravesar la fase de independencia. Influenciados generalmente por pares, hacen uso de sustancia de manera ocasional y los adolescentes que presentan personalidad compleja pueden ser más sensibles a dependencia de drogas $(18,19)$.

Por sus características evolutivas, como la búsqueda de identidad personal e independencia, alejamiento de los valores familiares y énfasis en la necesidad de aceptación por el grupo de iguales, la adolescencia se convierte en la etapa evolutiva con mayor riesgo de inicio del consumo de drogas (20).

Los factores de riesgos son comprendidos en el estudio como cualquier circunstancia o evento de naturaleza biológica, psicológica o social, cuya presencia o ausencia modifica la probabilidad de que se presenten problemas determinados en una persona o comunidad.

Los factores de protección son aquellos factores psicológicos o sociales que modifican la acción de un factor de riesgo para desestimular o evitar la aparición de la problemática (21).

Los modelos explicativos de los factores de riesgo para el consumo de drogas han determinado factores de riesgo a nivel individual, familiar, grupo de pares, social, educativo. En especial, los factores de riesgo a nivel familiar están relacionados con la desorganización familiar, poca relación con la familia, presencia de problemas afectivos en el hogar, ausencia de los padres, presencia de problemas en el manejo de la familia, expectativas no claramente definidas en relación a las conductas esperadas, falta de control, disciplina inconsistente o dura, conflictos matrimoniales, aprobación del uso del alcohol o de la droga y abuso 
del tabaco, el alcohol o de otras drogas por parte de los padres (21).

La familia es el primer elemento socializador, en cuyo seno se educa y forma al individuo, para que sus miembros sean capaces de desarrollarse y ser personas autónomas, que puedan enfrentarse e integrarse a la vida. De esta manera, los factores familiares se relacionan con los estilos parentales de manejo familiar, incluyendo el tipo de comunicación construida, el establecimiento de normas y el manejo de sanciones, el clima emocional familiar y la cultura en torno a las drogas $(22,23)$.

Considerando que los adolescentes están inmersos en un sinnúmero de factores de riesgos (biológicos, ambientales, de comportamiento, socio-culturales, económicos), que aumentan la probabilidad de que se inicien en el consumo de drogas, y, por consiguiente, acaben teniendo problemas con dichas sustâncias (24), este estudio presenta como objetivo describir el consumo de drogas y los factores de riesgo familiar en los adolescentes del Reparto Primero de Mayo de la ciudad de León, Nicaragua.

La identificación de estos factores puede ofrecer subsidios importantes para la formulación de programas de prevención e intervención para los adolescentes relacionados al uso y abuso de drogas psicoactivas.

\section{MATERIALES Y MÉTODOS}

Es un estudio de enfoque cuantitativo, de tipo descriptivo y transversal, ya que los sujetos fueron evaluados una única vez. El escenario fue el Reparto Primero de Mayo de la ciudad de León, Nicaragua.

El Reparto está ubicado en el occidente del país, específicamente en zonas marginales de la ciudad de León. Limita al este con el Reparto Venceremos y la carretera Bypaas, al Oestes con la segunda Etapa del Reparto primero de mayo, al Norte con Reparto José Benito Escobar y al oeste con el Reparto Aracelis Perez. El Reparto Primero de Mayo está dividido en tres etapas, habitando en su mayoría una población vulnerable expuesta a diferentes factores de riesgos y a escasos factores protectores, que conllevan a situaciones de peligros especialmente a los adolescentes y jóvenes.

La población de estúdio era formada por 258 adolescentes que habitan en la tercera etapa del Reparto Primero de Mayo entre 15 a 19 años de edad. La muestra del estudio fue formada por 76 adolescentes que atendieron a los criterios de inclusión: adolescentes entre 15 y 19 años de edad, que viven en la tercera etapa del Reparto, de de ambos los sexos y que concordaron en participar del estudio.
El tipo de muestreo utilizado fue el método probabilístico aleatorio simple, para que toda la población en estudio tuviera la misma probabilidad de ser elegida, hasta obtener la muestra de 76 jóvenes que corresponde al $30 \%$ de la población en estudio.

Para la recolección de datos se utilizaron dos instrumentos: un cuestionario sócio demográfico con informaciones sobre edad, sexo, escolaridad, se estudia actualmente, estado civil de los padres y consumo de alcohol y otras drogas y un instrumento diseñado a través de preguntas cerradas. Para la elaboración del instrumento se realizó revisión de bibliografía relacionada con las variables: factores de riesgo familiar y consumo de drogas por adolescentes.

Las preguntas del instrumento fueron divididas de acuerdo a los siguientes dominios: ausencia de los familiares, presencia de problemas afectivos en el hogar, presencia de problemas en el manejo de la familia, expectativa no claramente definida en relación a las conductas esperadas, relaciones conflictivas y actitud de la familia ante el consumo de alcohol y otras drogas.

El instrumento se consultó con 2 investigadores familiarizados con el tema y las variables a medir, sugiriendo correcciones en algunas preguntas. Se realizaron las correcciones para la aplicación de la prueba piloto.

Se realizaron 2 pruebas piloto (test) a jóvenes con las mismas características del Reparto Primero de Mayo. Se les explicó el objetivo del cuestionario, que no tendría ninguna consecuencia negativa y que la participación era voluntaria. En un primer momento se realizó a 6 jóvenes, observándose algunas dificultades de comprensión en algunos itenes. Se realizaron las modificaciones y se hizo una segunda prueba piloto a 12 jóvenes del mismo reparto, a los mismos 6 anteriores y 6 jóvenes más ( 7 varones y 5 mujeres) obteniendo resultados positivos y dando por aprobado el instrumento para la aplicación en el estudio.

Los participantes fueron recrutados a partir de las informaciones obtenidas del censo realizado por el Puesto de Salud. La recolección de datos fue realizada mediante el contacto directo con los adolescentes a través de una encuesta, en local reservado, com fecha y horário previamente agendado de acuerdo con la disponibilidad. Los participantes aceptaram participar de la investigación y firmaron el Término de Consentimiento Libre y Escolarecido, después de recibiren informaciones sobre la naturaleza y los objetivos de la investigación. Los cuestionários fueron aplicados individualmente y en un único encuentro. 
Para el tratamiento de la información se desarrolló una base de datos con cada una de las interrogantes de los instrumentos en el programa EPIINFO. Se utilizó estadística descriptiva: frecuencias y porcentajes en las variables.

El presente trabajo de investigación fue regido en su parte ética por las normas para la investigación en salud estipuladas en la Resolución 008430 de octubre 4 de 1993. La investigación fue sometida al Comité de Ética en Investigaciones Biomédicas (CIEB,) de la Universidad Nacional Autónoma de Nicaragua León Nicaragua, aprobado para su debida aplicación con el número: FWA00004523/IR00003342 en acta no 21.

Para esta investigación se tuvo en cuenta los principios éticos universales de beneficencia, no maleficencia, veracidad, autonomía y fidelidad, también, fue preservada la privacidad, confidencialidad y respeto a la dignidad humana.

\section{RESULTADOS}

Características sociodemográficas de los adolescentes participantes del estudio.

Los adolescentes encuestados, $46(61 \%)$ están en las edades de 18 a 19 años, $65 \%$ son del sexo masculino, $43 \%$ tienen nivel secundaria incompleta, $51 \%$ no estudian actualmente, $60 \%$ no trabaja, $88 \%$ son solteros, $67 \%$ católicos, $42 \%$ son hijos menores y $43 \%$ de los padres son separados (Tabla 1).

TABLA 1. Caracterización sociodemográfica de los estudiantes (n=76). Nicaragua-León, 2010.

\begin{tabular}{|ll|}
\hline Variable & $\mathbf{n}^{\mathbf{0}}(\mathbf{\%})$ \\
\hline Grupo etário (años) & $30(39)$ \\
\hline $15-17$ & $46(61)$ \\
\hline Sexo & \\
\hline Femenino & $27(35)$ \\
Masculino & $49(65)$ \\
Escolaridad & \\
\hline Primaria incompleta & $25(33)$ \\
\hline Primaria completa & $9(12)$ \\
\hline Secundaria incompleta & $33(43)$ \\
Secundaria completa & $3(4)$ \\
\hline Universidad & $6(8)$ \\
\hline Estudia actualmente & \\
\hline Si & $37(49)$ \\
\hline No & $39(51)$ \\
\hline Trabaja & \\
\hline Si & $30(40 \%)$ \\
\hline No & $46(60 \%)$ \\
\hline Estado civil adolescentes & $6(8)$ \\
\hline Acompañados & $3(4)$ \\
\hline Casados & $67(88)$ \\
\hline Solteros & \\
\hline
\end{tabular}




\begin{tabular}{|ll|}
\hline Religión & \\
\hline Católico & $51(67)$ \\
\hline Evangélico & $15(20)$ \\
\hline Outro & $10(13)$ \\
\hline Lugar família & \\
\hline Hijo Mayor & $24(31)$ \\
Hijo Menor & $32(42)$ \\
\hline Hijo Único & $2(3)$ \\
\hline Outro & $8(24)$ \\
\hline Estado civil de los padres & \\
\hline Casados & $16(21)$ \\
\hline Separados & $33(43)$ \\
\hline Unión Libre & $27(36)$ \\
\hline
\end{tabular}

Fuente: Datos de la investigación

En tabla 2 se refleja el consumo de sustancias psicoactivas por parte de los adolescentes participantes del estudio, 40 (53\%) de los adolescentes hacían uso de alcohol y tabaco e 36 (47\%) consumian otras drogas.

\section{TABLA 2. Consumo de drogas por los adolescentes del estudio (n=76). Nicaragua-León, 2010.}

\begin{tabular}{|ll|}
\hline Variable & $\mathbf{n}^{\mathbf{0}}(\%)$ \\
\hline Consumo de alcohol y tabaco & $40(53)$ \\
\hline Consumo de otras drogas & $36(47)$ \\
\hline
\end{tabular}

\section{Fuente: Datos de la investigación}

\section{Factores de riesgo familiares}

\section{Ausencia de los familiares}

Los resultados demuestran que $65 \%$ de los adolescentes conviven con diferentes parentescos familiares y $35 \%$ con los padres y hermanos. Los adolescentes pasan la mayor parte del tiempo de sus vidas con diferentes parentescos familiares, comparado al tiempo que conviven con sus padres y hermanos.

Cabe destacar que un $61 \%$ de los padres de familia han estado fuera del hogar desde 1 año hasta por más de 7 años, buscando el sustento de su familia, lo que hace que estos adolescentes tengan que convivir con algún integrante del resto de la familia.

\section{Presencia de problemas afectivos en el hogar}

En la tabla 3 se observa que en un $51 \%$ de los adolescentes manifiestan nunca sentir en algún grado de magnitud el sentimiento de abandono. 
TABLA 3. Sentimiento de abandono por parte de los adolescentes (n=76). Nicaragua - León, 2010.

\begin{tabular}{|lc|}
\hline Variable & $\mathbf{n}^{\mathbf{0}}(\mathbf{\%})$ \\
\hline Nunca & $39(51)$ \\
A veces & $27(35)$ \\
Casi siempre & $8(11)$ \\
Siempre & $2(3)$ \\
\hline
\end{tabular}

Fuente: Datos de la investigación

De esta forma, $49 \%$ de los adolescentes considera que su familia siempre le brinda amor y seguridad, $45 \%$ le brinda a veces o casi siempre y solamente $6 \%$ nunca le brinda amor y seguridad.

\section{Expectativas no claramente definidas en relación a las conductas esperadas}

Otro aspecto importante que se encontró en el estudio es que 50\% de los padres de familia tienen comportamiento de autoridad y afecto, el $23 \%$ son excesivamente rígidos y $27 \%$ tienen un comportamiento permisivo y desinteresado. Para $74 \%$ de los adolescentes, sus padres o tutores hacen llamado de atención con respeto y en privado, para el $17 \%$ hacen llamados de atención humillantes y en público y para un $9 \%$ ningún llamado de atención.

\section{Presencia de problemas en el manejo de la familia}

En la tabla 4 se aprecia que la mayoría de los adolescentes $(35 \%)$ se sienten seguros apenas algunas veces para buscar ayuda de sus padres o tutores.

TABLA 4. Sentimiento de los adolescentes en cuanto a la posibilidad de buscar ayuda en sus padres o tutores (n=76). Nicaragua - León, 2010.

\begin{tabular}{lc|} 
Variable & $\mathbf{n}^{\mathbf{0}}(\%)$ \\
\hline Nunca & $18(24)$ \\
A veces & $27(35)$ \\
Casi siempre & $9(12)$ \\
Siempre & $22(29)$ \\
\hline
\end{tabular}

Fuente: Datos de la investigación

\section{Relaciones Conflictuosas}

La tabla 5 está relacionada a la presencia de discusión en la familia de los adolescentes y $78 \%$ de los sujetos relatan discusión con otros miembros familiares, pero no con sus padres o hermanos.

TABLA 5. Existencia de discusión en la familia de los adolescentes (n=76). Nicaragua - León, 2010.

\begin{tabular}{lc|}
\hline Variable & $\mathbf{n}^{\circ}(\%)$ \\
\hline Discusión entre los padres & $0(0)$ \\
Discusión con usted y su papá & $0(0)$ \\
Discusión con usted y su mamá & $0(0)$ \\
Discusión con usted y sus hermanos & $0(0)$ \\
Discusión en la familia o con otros & $59(78)$ \\
No existe discusión & $17(22)$ \\
\hline
\end{tabular}

Fuente: Datos de la investigación 
Para los adolescentes participantes del estudio, 79\% no recibe algún tipo de violencia por parte de alguien de su familia, $8 \%$ sufren abuso físico y $13 \%$ abuso psicológico por parte de alguien de su familia.

\section{Actitud de la familia ante al consumo de substancias psicoactivas}

Los resultados demuestran que $87 \%$ de los adolescentes participantes relatan que su familia les prohíbe al consumo de alcohol y tabaco, $8 \%$ presenta actitud permisiva y $5 \%$ indiferencia. En relación al consumo de marihuana, cocaína, e inhalantes, $83 \%$ de los familiares tienen actitud de prohibiciones, $8 \%$ actitud permisiva e $9 \%$ indiferencia.

Cabe resaltar que de acuerdo a los adolescentes, $62 \%$ de sus padres no consumen alcohol, tabaco o algún tipo de drogas. Entre los que consumen, la mayoría (37\%) usa alcohol y tabaco.

\section{DISCUSIÓN}

La mayoría de los adolescentes están entre las edades de 18 y 19 años y con estudios no concluidos de acuerdo a sus edades, por lo que estos adolescentes no tienen aspiraciones positivas en su vida y carecen de visión de futuro. Estudios hacen referencia a que los adolescentes a esta edad atraviesan por un período crítico, de cambios físicos y psicosociales, que los puede condicionar a diversos riesgos (24).

Las drogas más consumidas por los adolescentes del estudio fueron el alcohol y tabaco. Sin embargo, 47\% mencionaron uso de otras drogas. La adolescencia es el grupo etario con mayor preocupación cuanto al consumo de drogas, pues los años adolescentes constituyen un período de exposición a las drogas y de vulnerabilidad, caracterizada por cambios que afectan tanto los aspectos físicos, cognitivos cuanto sexuales (18-20).

En cuanto a la convivencia de los adolescentes en la familia, en su mayoría tienen familias disfuncionales, lo que trae conflictos internos en los adolescentes. Esta situación se describe en la literatura como un factor de riesgo, ya que cuando los adolescentes conviven con personas de diferente parentesco o familias monoparentales, la estructura del núcleo familiar se desintegra y puede traer costos emocionales y de comportamiento al querer adaptarse a nuevas normas, ocasionando enormes implicaciones sociales como escape a la tensión que éstos experimentan (21-26).

De esta forma, la disfunción familiar es un factor predisponente para el desarrollo de conductas poco saludables en los adolescentes y buscar un escape a la falta de adaptación en el consumo de sustancias dañinas para su salud.

Los adolescentes necesitan cariño, afecto y apoyo por parte de sus padres; así como también comprensión y paciencia, ya que están sufriendo una serie de cambios en su forma de pensar y en su aspecto físico, que en un primer momento, no saben como afrontar y por lo tanto necesitan de la ayuda de los adultos. El sentimiento de abandono y falta de amor y seguridad hacen que éstos estén en peligro de perder su bienestar físico y psicoemocional, así como también su capacidad de seguridad en sí mismos, el cual puede traer como consecuencia que algunos adolescentes recurran a conductas autodestructivas como el consumo de alcohol, droga y por consecuente en crisis y depresión(21-27).

Sin embargo, la presencia de problemas afectivos en el hogar se ha considerada como un factor de riesgo familiar, en este estudio, la mayoría de los adolescentes no presentaron sentimientos de abandono y sienten que su familia les brinda amor y seguridad.

En cuanto a las expectativas no claramente definidas en relación a las conductas esperadas, los datos del estudio demuestran que la mayoría de los adolescentes consideran que sus padres les tratan con autoridad y afecto, al igual que les llaman la atención con respeto y privado. Siendo así, para estos adolescentes que existen reglas definidas en cuanto a sus conductas esperadas.

Según el estudio, son pocos los adolescentes que buscan ayuda en sus padres al tener alguna duda o problema. El débil establecimiento de un vínculo afectivo con los/as hijos/as pueden volverse en riesgo para el consumo de drogas, ya que muchos de estos adolescentes se sienten obligados a buscar fuera del hogar a personas que los escuchan y los comprenden pero que también los puedan llevar a conductas de riesgo. En este sentido, estudios realizados (22-28), han puesto de manifiesto como primer factor negativo del clima familiar la presencia de problemas en el manejo de la familia, con la ausencia de diálogo afectivo y en consecuencia, la necesidad de un ambiente que fomente este diálogo como condición indispensable para la convivencia, el respeto y el desarrollo de capacidades, tales como la posibilidad de hablar y exponer los puntos de vista respecto a las drogas u otros temas de interés con sus padres.

En la mayoría de los adolescentes opinan que existen relaciones conflictivas en la familia, especialmente por medio de discusiones. Estudios realizados. consideran que los factores de riesgo relacionados con las inconsistentes prácticas en el manejo familiar; conflicto familiar y bajo apego a la familia llevan a unas prácticas de crianza pobre y a veces desordenada o poco consistente (28-30). Cuando hay una situación 
de conflictos familiares se incrementa el riesgo tanto para conductas delictivas como para conductas de uso de drogas.

En cuanto a las relaciones conflictivas, se debe resaltar que los adolescentes participantes no reciben, en su mayoría, violencia por parte de alguien de su familia.

La actitud de la familia ante el consumo de alcohol y otras drogas, se observó la existencia de prohibiciones con relación a las drogas lícitas como alcohol y tabaco y cuanto el consumo de marihuana, cocaína e inhalantes.

En relación a los padres, la mayoría no consume drogas lícitas o ilícitas. Esta no es la realidad observada con relación a los adolescentes participantes de la investigación, una vez que el $53 \%$ de los adolescentes participantes de la investigación consumen alcohol y tabaco y el $47 \%$ consumen algún tipo de drogas e iniciando el consumo a muy temprana edad, con una media de 14 años de edad. El joven que busca encontrar su propia identidad, muchas veces ve en la droga una manera de distinguirse en medio de otros adolescentes, y con esto encuentra también la manera de hacer amigos, quienes, buscando una identidad de grupo, repiten actitudes y comportamientos sin tener aún la capacidad de medir lo que serán las consecuencias a largo plazo (30,31).

\section{CONCLUSIONES}

En este estudio fueron identificados el consumo de drogas y los factores de riesgo familiar en los adolescentes del Reparto Primero de Mayo de la ciudad de León, Nicaragua. Los factores de riesgo familiares fueron asociados a la ausencia de los padres, caracterizada por la disfunción familiar; presencia de problemas afectivos en el hogar; presencia de problemas en el manejo de la familia; expectativa no claramente definida en relación a las conductas esperadas; relaciones conflictivas y actitud de la familia ante el consumo de alcohol y otras drogas.

De esta forma, se concluye que el fenómeno de las drogas es multifactorial con consecuencias para la salud, la educación y la seguridad pública, considerando su prevalencia en los adolescentes.

Para el personal de enfermería, conocer los factores de riesgo familiares presentes en el consumo de drogas contribuye a la planificación y ejecución de planes y/o programas preventivos en relación al uso de sustancia psicoactivas, en conjunto con diferentes actores sociales. La familia es fuente de protección, afecto y seguridad para sus miembros. Es un espacio privilegiado para la socialización primaria, de modo que un ambiente seguro, transmite a los adolescentes sus normas y valores.

Es necesario nuevos estudios en esta temática con mayor análisis, que permitan continuar profundizando sobre el fenómeno de las drogas en este grupo vulnerable y desarrollar estrategias que contribuyan a la efectiva inserción social de los adolescentes.

\section{AGRADECIMIENTOS}

Agradecemos a la Comisión Interamericana para el Control del Abuso de Drogas/CICAD de la Secretaria de Seguridad Multidimensional/SSM de la Organización de los Estados Americanos/OEA, la Secretaria Nacional de Políticas sobre Drogas/SENAD del Gabinete de Seguridad Institucional/Brasil, la Escuela de Enfermería de Ribeirao Preto de la Universidad de Sao Paulo y Centro Colaborador de la Organización Mundial de la Salud para el Desarrollo de la Investigación en Enfermería, la población representada en los estudios de investigación, bien como a las autoridades de las universidades representadas por los participantes del Programa On-line de Especialización en Investigación sobre el Fenómeno de las Drogas - PREINVEST, periodos 2009 y 2010.

\section{REFERENCIAS BIBLIOGRÁFICAS}

1. Comisión Interamericana para el Control del Abuso de Drogas - CICAD. Estrategia Hemisférica sobre Drogas. Secretaría de Seguridad Multidimensional. Washington DC, 2010: 1-7. Disponible en: http://www.cicad.oas.org/ apps/Document.aspx?Id=954 Consultado: Marzo 4, 2010.

2. Comisión Interamericana para el Control del Abuso de Drogas - CICAD/Organización Mundial de la salud OMS. Neurociencia del consumo y dependencia de sustancias psicoactivas. Washington DC, 2005. Disponible en: http://www.cicad.oas.org/Main/default_SPA.asp Consultado: Abril 1, 2010.

3. Comisión Interamericana para el Control del Abuso de Drogas - CICAD. Proyecto de la CICAD de las Escuelas de Enfermeria sobre Prevención del Uso y Abuso de Drogas, Integración Social y Promoción de la Salud en América Latina. Washington DC. Disponible en: http://www.cicad.oas.org/Reduccion_Demanda/esp/ Enfermeras/Peru/PeruIII.htm Consultado: Febrero 8, 2010.

4. Oficina de las Naciones Unidas sobre drogas y delito - UNODC. Consumo de drogas y nuevas tendencias en reducción de la demanda. Oficial. Colombia, 2006. Disponible en: http://www.descentralizadrogas.gov.co/ Consultado: Marzo 6,2010. 
5. Zárate M, Zavaleta A, Danjoy D, Chanamé E. Prácticas de consumo de tabaco y otras drogas en estudiantes de ciencias de la salud de una universidad privada de Lima, Perú. Revista Investigación y Educación en Enfermería. Medellín. 2006; 24: 72-81.

6. Figueroa SDS, Cunningham J, Strike C, Brands B, Wright MGM. Normas percibidas por los estudiantes universitarios hondureños acerca de sus pares y el uso de tabaco, alcohol, marihuana y cocaína. Rev. Latino-Am. Enfermagem [online]. 2009; 17 (n.spe): 851-857.

7. Wright MGM. La contribución de la Enfermería frente al fenómeno de las drogas y la violencia en América Latina: Un proceso de construcción. Cienc Enferm. Rev Iberoam Invest. 2002; 8(2): 9-19.

8. Castro N, Cortés P, Vasters GP, Costa Jr. ML. Uso de drogas entre estudiantes de la Facultad de Ciencias Médicas de la Universidad Nacional Autónoma de Nicaragua, León, Nicaragua. Rev. Latino-Am. Enfermagem. 2010; 18 : 606-12.

9. Dalri RCMB. Tabagismo entre trabalhadores de uma usina sucro-alcooleira no brasil. Cienc. Enferm. 2006; 12(1):53-60.

10. Herrera-Rodríguez A, Prieto-Silva R, Veloza-Gomez M, Riquelme-Hernández G, Nóbrega MPSS, Mitchell C. Policonsumo simultáneo de drogas en estudiantes de facultades de ciencias de la salud/ciencias médicas en siete universidades de cinco países de América Latina y en un país del Caribe: implicaciones de género, legales y sociales. Texto Contexto Enferm. 2012; 21 (Esp): 17-24.

11. García-Baquero M, Tobon-Borrero L, Ramirez-Duarte M. Consumo de substancias psicoactivas en estudiantes Universitarios en Villavicencio 2009-2010. Revista Cuidarte. 2011; 2(2): 161-7.

12. Ministerio de Educación - MINED. Reporte del periódico: El Nuevo Diario. "Bañados", es la nueva droga que está consumiéndose en las escuelas de Managua, 2005.

13. Muñoz-Pavón M. Prevalencia del consumo de drogas en los casos atendidos por lesiones y necropsias realizadas en el instituto de medicina legal en el segundo semestre. Universidad Nacional Autónoma de Nicaragua. Managua, 2006.

14. Vilela MV, Ventura CAA, Silva EC. Conocimientos de estudiantes de enfermería sobre alcohol y drogas. Rev. Latino-Am. Enfermagem [online]. 2010; 18 (n.spe): 529-534.

15. Leiva-Díaz V, Vasters GP, Costa JR ML. Caracterización de estudiantes de la carrera de enfermería sobre consumo de drogas lícitas e ilícitas. Rev. Latino-Am. Enfermagem [online]. 2010; 18 (n.spe): 565-572.

16. Wright MGM. La contribución de la enfermeria frente al fenómeno de las drogas y la violencia en América Latina: un proceso de construcción. Cienc. Enferm. 2002; 8 (2): 09-19.

17. León A. Entre dos monedas. Dianova nicaragua. Boletin Trimestral Diciembre 2008. Disponible en: http://www. pnsd.msc.es/Categoria2/publica/pdf/memo2000.pdf Consultado: Septiembre 4, 2009.

18. Juventud y las naciones unidas. Informe sobre la juventud mundial: Uso indebido de drogas, 2005. Disponible en: http://www.cinu.mx/minisitio/UNjuventud/docs/A_60_61.pdf Consultado: Septiembre 6,2009.

19. Facundo FRG, Pedrão LJ. Fatores de risco pessoais e interpessoais no consumo de drogas ilícitas em adolescentes e jovens marginais de bandos juvenis. Rev. Latino-Am. Enfermagem. 2008; 16(3): 368-374.

20. Navarro de Sáez M, Cometto MC, Aespig H, Cromaguera F, Gómez P, Cerró-Ruza E. Relación entre factores de riesgo del consumo de drogas y pronóstico académico en estudiantes de nuevo ingreso en la carrera de enfermería en dos universidades latinoamericanas. Enferm. Glob. [online]. 2009; (17): 0-0.

21. Ugarte RM. Factores de riesgo. La familia como factores de riesgo, protección y resiliencia en la prevención del abuso de drogas en adolescentes. Capitulo V (En línea). Lima: 2001. Diponible en: http://www.venumperu.com/ libro2.htm Consultado: Abril 2, 2010.

22. Lobato CM. Consumo de drogas en la adolescencia. Factores de riesgo y protección asociados. (En Línea). Departamento de Menores Ministerio de Justicia. Julio, 2007. Disponible en: http://justiciapenaladolescente. blogspot.com/ Consultado: Octubre 10, 2009.

23. Álvarez-Aguirre A, Alonso-Castillo MM, Zanetti ACG. Consumo de alcohol y autoestima en adolescentes. Rev. Latino-Am. Enfermagem [online]. 2010; 18 (n.spe): 634-640.

24. Sussman S, Unger JB, Dent CW. Peer group self-identification among alternative high school youth: A predictor of their psychosocial functioning five years later. Int J Clin Health Psychol. 2004; 4(1): 9-25.

25. Vásquez-Zarate V, Nava-Esquivel C, Valle-Flores R. Grupo de trabajo de PEMEX. Dirección, subdirección de servicios de salud corporativa de administración Promoción a la salud en adolescente. Coordinación Nacional de Activación Física y Tiempo Libre. Disponible en: http://www.serviciosmedicos.pemex.com/salud/adolescencia. pdf pag 1-5 17. Acceso 5 abril de 2010.

26. Ventura CAA, Brands B, Adlaf E, Giesbrecth N, Simich L, Wright MGM, et al. Políticas e leis sobre drogas ilícitas no Brasil e a perspectiva de familiares e pessoas próximas a usuários de drogas: estudo na Cidade de Ribeirão Preto, São Paulo, Brasil. Rev. Latino-Am. Enfermagem [online]. 2009; vol 17 (n.spe): 810-816. 
27.Laespada T, Iraurgi L, Arostegui E. Factores de riesgo y protección frente al consumo de drogas en jóvenes: Hacia un modelo explicativo frente al consumo de drogas. Universidad de Deusto. IDD. Julio, 2004 Disponible en: http://www.izenpe.com/s15-4812/es/contenidos/informacion/publicaciones_ovd_inf_txostena/es_9033/ adjuntos/informe_txostena14.pdf Acceso 6 abril de 2010.

28. Castro SME. Factores de protección asociados al riesgo del consumo de sustancias adictivas en población de jóvenes estudiantes. En Tapia, R. (coord.), Las adicciones: Dimensión, impacto y perspectivas. 2nd ed. México: El Manual Moderno; 2001. p. 277-289.

29. Rellánez J, Diaz B, Wagner F, Pérez V. Factores psicosociales asociados con el abuso y la dependencia de drogas entre adolescentes: Análisis bivariados de un estudio de casos y controles. Salud Mental. 2004; 27(3): 54-64.

30. González SJD, García AVR, Córdova A. Uso de sustancias entre adolescentes y su asociación con síntomas de depresión y percepción de sus relaciones familiares, Centros de Integración Juvenil, Informe de Investigación. México, 2004.

31. Becoña IE. Bases científicas de la prevención de las drogodependencias (EN CD ROM) Universidad de Santiago de Compostela, edita: Ministerio del Interior, delegación del gobierno para el plan nacional sobre drogas secretaría general técnica. Madrid, 2002, p. 189-255. 